EPJ Web of Conferences 37, 01026 (2012)

DOI: $10.1051 /$ epjconf/20123701026

(C) Owned by the authors, published by EDP Sciences, 2012

\title{
GlueX: Photoproduction of Hybrid Mesons
}

\author{
Elton S. Smith ${ }^{\mathrm{a}}$ for the GlueX Collaboration \\ Thomas Jefferson National Accelerator Facility, Newport News, VA 23606 USA
}

\begin{abstract}
The goal of the GlueX experiment [1] is to provide critical data to help understand the soft gluonic field responsible for binding quarks in hadrons. Hybrid mesons, and in particular exotic hybrid mesons, provide the ideal laboratory for testing QCD in the confinement regime since these mesons explicitly manifest the gluonic degrees of freedom. Photoproduction is expected to be particularly effective in producing exotic hybrids but there is little data on the photoproduction of light mesons. GlueX will use the new $12-\mathrm{GeV}$ electron beam to produce a $9-\mathrm{GeV}$ beam of linearly polarized photons using the technique of coherent bremsstrahlung. A solenoid-based hermetic detector is under construction, which will be used to collect data on meson production and decays. These data will also be used to study the spectrum of conventional mesons, including the poorly understood excited vector mesons. This talk will describe the latest theoretical developments [2] to help understand how the data of hybrid mesons can provide insights into the fundamental theory of strong interactions.
\end{abstract}

\section{Introduction and background}

Quark degrees of freedom are displayed explicitly in the known hadron spectrum. But the gluon fields that confine the quarks inside hadrons have been shy to manifest their presence in the static properties of the measured spectrum. These gluonic degrees of freedom and interactions that are present in the QCD Lagrangian, are expected to give rise to many states in the spectrum with unique signatures. One class of such states is the hybrid meson, which can be naively thought of as a quark anti-quark pair coupled to a valence gluon $(q \bar{q} g)$. A subset of these hybrids have an unmistakable experimental signature: angular momentum $(J)$, parity $(P)$, and charge conjugation $(C)$ that cannot be created from just a quark-antiquark pair. Such states are called exotic hybrid mesons. A recent review of the status of these states can be found in reference [3]. The primary goal of the GlueX experiment in Hall D at Jefferson Lab is to search for and study these mesons.

Recently there have been giant steps forward in calculations of QCD on the lattice to compute and understand the meson spectrum. This numerical approach to QCD considers the theory on a finite, discrete grid of points in a manner that would become exact if the lattice spacing were taken to zero. The spectrum of eigenstates of QCD can be extracted from correlation functions of the type $\left\langle 0\left|O_{f}(t) O_{i}^{\dagger}(0)\right| 0\right\rangle$, where the $O^{\dagger}$ are composite QCD operators capable of interpolating a meson or baryon state from the vacuum. The time-evolution of the Euclidean correlator indicates the mass spectrum $\left(e^{-m_{n} t}\right)$ and information about quark-gluon substructure can be inferred from matrix-elements $\left\langle\mathrm{n}\left|O^{\dagger}\right| 0\right\rangle$. In a series of recent papers [2,4-6], the Hadron Spectrum Collaboration has explored the spectrum of mesons and baryons using a large basis of composite QCD interpolating fields, extracting a spectrum of states of determined $J^{P C}$, including states of high internal excitation.

As shown in Fig. 1, for the first time these calculations show a clear and detailed spectrum of exotic $J^{P C}$ mesons, with a lightest $1^{-+}$lying a few hundred $\mathrm{MeV}$ below a $0^{+-}$and two $2^{+-}$states. Beyond this, through analysis of the matrix elements $\left\langle\mathrm{n}\left|O^{\dagger}\right| 0\right\rangle$ for a range of different quark-gluon constructions, $O$, we can infer [2] that although the bulk of the non-exotic $J^{P C}$ spectrum has the expected systematics of

\footnotetext{
a e-mail: elton@jlab.org
}

This is an Open Access article distributed under the terms of the Creative Commons Attribution License 2.0, which permits unrestricted use, distribution, and reproduction in any medium, provided the original work is properly cited. 


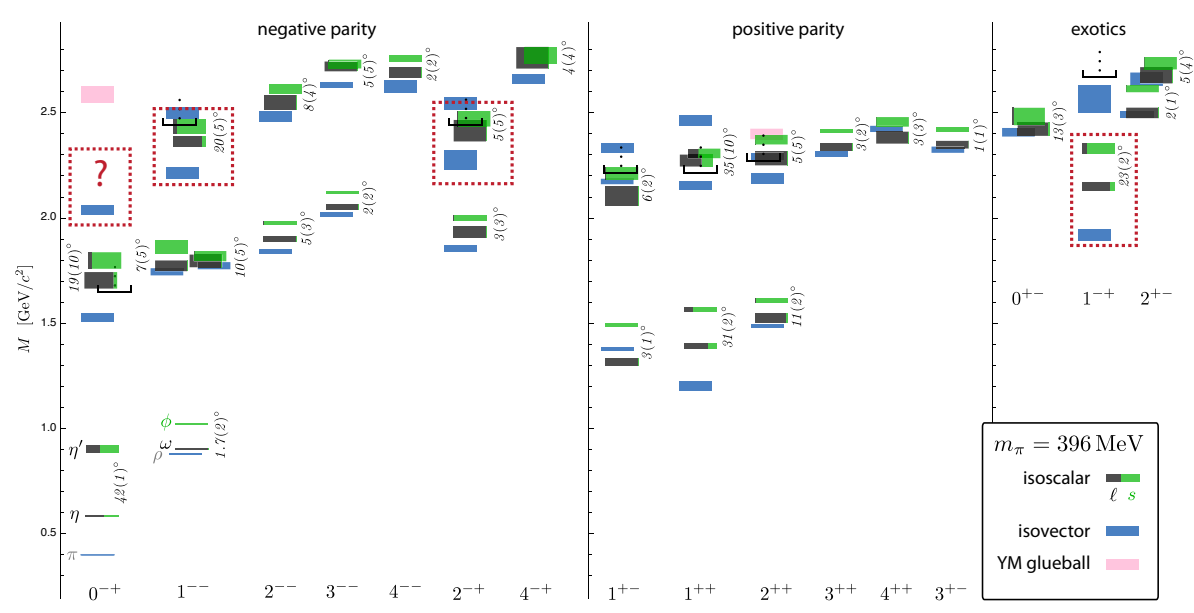

Fig. 1. A compilation of recent lattice QCD computations for both the isoscalar and isovector light mesons from Ref. [2], including $\ell \bar{\ell}(|\ell \bar{\ell}\rangle \equiv(|u \bar{u}\rangle+|d \bar{d}\rangle) / \sqrt{2})$ and $s \bar{s}$ mixing angles (indicated in degrees). The dynamical computation is carried out with two flavors of quarks, light $(\ell)$ and strange $(s)$. The $s$ quark mass parameter is tuned to match physical $s \bar{s}$ masses, while the light quark mass parameters are heavier, giving a pion mass of $396 \mathrm{MeV}$. The black brackets with upward ellipses represent regions of the spectrum where present techniques make it difficult to extract additional states. The dotted boxes indicate states that are interpreted as the lightest hybrid multiplet the extraction of clear $0^{-+}$states in this region is difficult in practice.

a $q \bar{q}$ bound state system, some states are only interpolated strongly by operators featuring non-trivial gluonic constructions. One may interpret these states as non-exotic hybrid mesons, and, by combining them with the spectrum of exotics, it is then possible to isolate a lightest hybrid supermultiplet of $(0,1,2)^{-+}$and $1^{--}$states, roughly $1.3 \mathrm{GeV}$ heavier than the $\rho$ meson. The form of the operator that has strongest overlap onto these states has an $S$-wave $q \bar{q}$ pair in a color octet configuration and an exotic gluonic field in a color octet with $J_{g}^{P_{g} C_{g}}=1^{+-}$, a chromomagnetic configuration. The heavier $(0,2)^{+-}$ states, along with some positive parity non-exotic states, appear to correspond to a $P$-wave coupling of the $q \bar{q}$ pair to the same chromomagnetic gluonic excitation. Exotic hybrids are predicted only when the quarks have their spins aligned.

\section{Understanding the spectrum}

To gain a qualitative understanding of the lattice predictions, we review some of the models and see how their spectra match the lattice calculations. This is by no means an exhaustive review, but rather a discussion of some of the approaches that have been made to understand the non-trivial configurations of gluonic fields within hadrons. In addition the mass scales cannot be compared directly so only the relative mass shifts are considered. The flux tube model [7] assumes the gluonic degrees of freedom behave as flux tubes with $J_{g}^{P C}=1^{+-}$and $1^{-+}$and predicts a total of eight degenerate hybrids. Six of them have the same $\mathrm{J}^{P C}$ quantum numbers as the lattice calculations, plus two additional ones with $\mathrm{J}^{P C}=1^{+-}$and $1^{++}$. All states are expected to have the same degenerate mass of approximately 1.9 $\mathrm{GeV}$. There is no evidence from the lattice for the $1^{-+}$gluonic configuration. Cotanch et al. use an effective Hamiltonian approach [8] and predict a total of seven states, four at $2.1 \mathrm{GeV}$ and three at higher mass. The only state that matches the lattice calculation quantum numbers is a single $1^{-+}$ exotic, so this model fails completely to reproduce the lattice spectrum. Guo et al. used a Coulomb gauge approach for heavy quarks and a constituent gluon with quantum numbers of $J_{g}^{C P}=1^{+-}$[9]. This model predicts the same quantum numbers as the lattice and a similar excitation spectrum, and it 
therefore could provide insight into the configuration of gluonic fields inside mesons. In particular the model predicts two $2^{+-}$exotic states, which are also seen in the calculated lattice spectrum.

\section{Photoproduction}

There is little data on meson photoproduction in the energy range of about $9 \mathrm{GeV}$. Almost all of what is known comes from bubble chamber measurements at SLAC. In particular, very little is known about reactions with multiple neutrals, which make up about $70 \%$ of the total cross section. Therefore, this regime provides a long list of unexplored reactions with discovery potential.

Estimates of the cross sections can be made starting from radiative transitions of hybrid mesons. The radiative decay widths of charmed mesons have been computed on the lattice by Dudek and collaborators with relatively good agreement to experiment [10]. The charmed radiative widths range from $100 \mathrm{keV}$ to $300 \mathrm{keV}$. The authors also compute the radiative decay of the $1^{-+} \eta_{c 1}$ exotic with a mass of $4.3 \mathrm{GeV}$ to $J / \psi \gamma$ to be $115 \pm 16 \mathrm{keV}$, which is of similar strength as the known charmed meson decays. Assuming that the photon couplings for light quark hybrids are similar, we can assume that hybrids would be produced in photoproduction at roughly the same rate as normal mesons. The lattice spectrum calculations suggest that exotic hybrids are built on top of quarks with spins aligned, which allows the production by photons to occur without any spin flip transitions.

In addition, linearly polarized photons increase sensitivity to resonance properties under certain circumstances. For a given produced resonance, linear polarization enables one to distinguish between naturalities of exchanged particles. If the production mechanism is known, linear polarization enables one to filter resonances of different naturalities.

\section{The GlueX Experiment}

The GlueX experiment will run in a new experimental area (Hall D) at Jefferson Lab, which has been built as part of the $12 \mathrm{GeV}$ Upgrade Project. The photon beam originates from coherent bremsstrahlung radiation produced by the $12 \mathrm{GeV}$ electron beam impinging on a $20 \mu \mathrm{m}$ diamond wafer. Orientation of the diamond and downstream collimation produces a photon beam peaked in energy around $9 \mathrm{GeV}$ with about $40 \%$ linear polarization. The photon energies and times are tagged with two detectors: a coarse tagger covering a broad energy range and a precision array for the coherent peak. A schematic view of the GlueX detector is shown in Fig. 2, which surrounds the hydrogen target. The solenoidal detector has tracking and calorimetry. Timing and triggering are aided by a forward time-of-flight wall and a thin scintillator that encloses the target. We briefly describe each of the detector components.

The GlueX detector is based on a $2.2 \mathrm{~T}$ superconducting solenoid, which provides the essential magnetic field for tracking. The solenoidal geometry also has the benefit of reducing electromagnetic backgrounds in the detectors since low energy $e^{+} e^{-}$pairs spiral within a small radius of the beamline. The field is provided by four superconducting coils, which have been tested individually and are currently being integrated with the cryogenic supply box and control system in preparation for testing of the assembled magnet.

Charged particle tracking is performed by two systems: a central straw-tube drift chamber (CDC) and four six-plane forward drift chamber (FDC) packages. The CDC [11] is composed of 28 layers of $1.5 \mathrm{~m}$-long straw tubes, with some of the layers at a $6^{\circ}$ stereo angle to supply $z$ measurements. Each FDC package is composed of six planes of anode wires. The cathode strips on either side of the anode cross at $\pm 75^{\circ}$ angles, providing a two-dimensional intersection point on each plane. The position resolutions of the CDC and FDC are about $150 \mu \mathrm{m}$ and $200 \mu \mathrm{m}$, respectively. Together the approximate momentum resolution is $1-3 \%$, averaged over the kinematical regions of interest.

Like tracking, the GlueX calorimetry system consists of two detectors: a barrel calorimeter [12] with cylindrical geometry (BCAL) and a forward lead-glass calorimeter with planar geometry (FCAL). The primary goal of these systems is to detect photons that can be used to reconstruct $\pi^{0}$ 's and $\eta$ 's, which are produced in the decays of heavier states. The BCAL is a relatively high-resolution sampling calorimeter, based on $1 \mathrm{~mm}$ double-clad Kuraray scintillating fibers embedded in a lead matrix. 


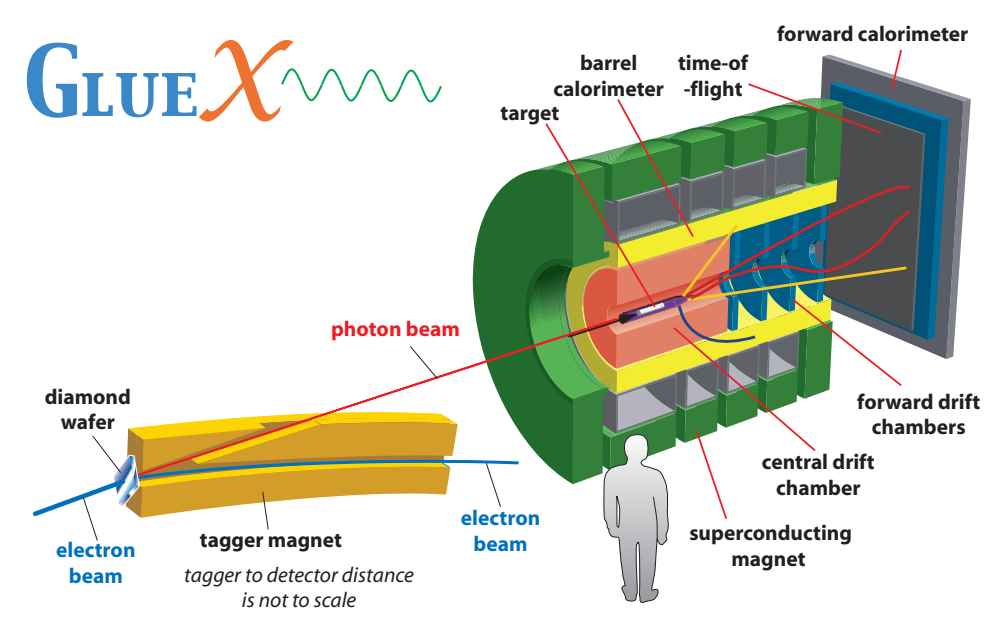

Fig. 2. A schematic of the GlueX detector and beam.

Modules are read out on each end by silicon photomultiplier (SiPM) arrays (Hamamatsu S12045(X) MPPC), which are unaffected by the high magnetic field of the GlueX solenoid flux return. The forward calorimeter is composed of 2800 lead glass modules, stacked in a circular array. Each bar is coupled to a conventional phototube, which are powered by custom-built Cockroft-Walton style bases [13] in order to reduce power dissipation and high voltage control system costs. The fractional energy resolution of the combined calorimetry system $\sigma(E) / E$ is approximately $5 \%-6 \% / \sqrt{E[\mathrm{GeV}]}$.

The existing particle identification capabilities are derived from several subsystems. A dedicated forward time-of-flight wall (TOF), which is constructed from two planes of $2.5 \mathrm{~cm}$-thick scintillator bars, provides about 70 ps timing resolution on forward-going tracks within about $10^{\circ}$ of the beam axis. This information is complemented by time-of-flight data from the BCAL and specific ionization $(d E / d x)$ measured with the CDC, both of which are particularly important for identifying the recoil proton in $\gamma p \rightarrow X p$ reactions. The combined PID system in the baseline design is sufficient for identification of most protons in the kinematic regions of interest for GlueX. However, the initial setup only allows for exclusive kaon identification for track momenta less than $2.0 \mathrm{GeV} / c$.

\section{Initial physics goals and sensitivity}

The GlueX physics program provides an excellent opportunity for both the study of conventional mesons and the search for exotic mesons in photoproduction. Reconstructable final states will initially be limited to those decaying into non-strange states: $\pi, \eta, \eta^{\prime}$, and $\omega$.

Table 1 summarizes the expected lowest mass exotics and possible decay modes. Initial searches will likely focus on the $\pi_{1}$ isovector triplet and the $\eta_{1}$ isoscalar. It will also be important to try to establish the other (non-exotic) members of the hybrid multiplet: the $0^{-+}, 1^{--}$, and $2^{-+}$states predicted by lattice calculations. One reaction of interest is $\gamma p \rightarrow \pi^{+} \pi^{-} \pi^{+} n$. The $(3 \pi)^{ \pm}$system has been studied extensively with data from E852 [14,15], COMPASS [16], and CLAS [17]. Most recently COMPASS has reported evidence for exotic $\pi_{1}(1600) \rightarrow \rho \pi$ decay. In an attempt to exercise our software framework, a full analysis of simulated GlueX data was performed with this channel. A GEANT-based simulation was developed to model all active and inactive material within the detector volume. Individual hits and background were simulated on all detector components, and reconstruction algorithms were utilized to reconstruct each event without knowledge of the true generated particles.

As a first step to evaluate the sensitivity to small amplitudes that is provided by the GlueX detector acceptance and resolution, we performed an amplitude analysis on a sample of purely generated $\gamma p \rightarrow$ $\pi^{+} \pi^{-} \pi^{+} n$ events. Several conventional resonances, the $a_{1}, \pi_{2}$, and $a_{2}$, were generated along with a small $(<2 \%)$ component of exotic $\pi_{1}$. The expectation, however, is that the exotic signal should be 


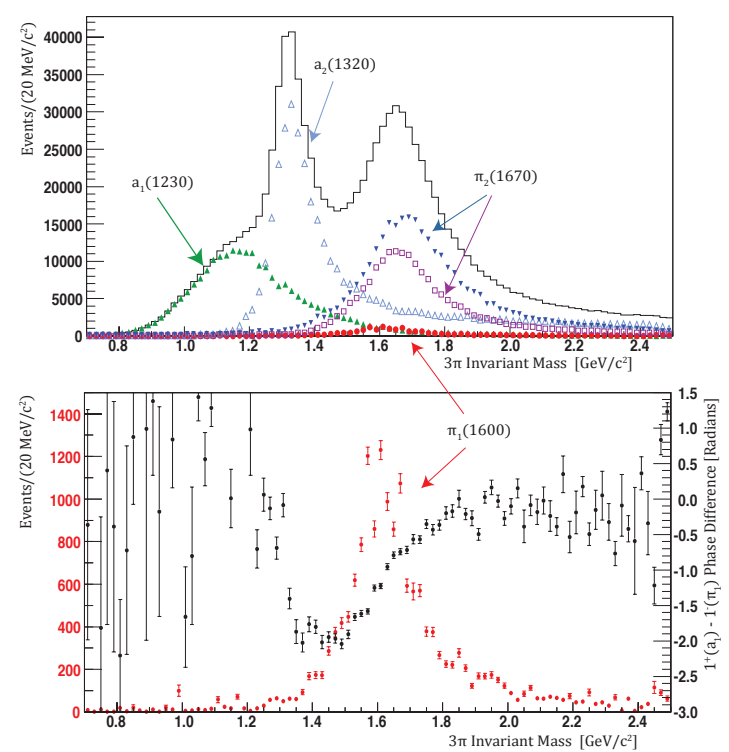

Fig. 3. A sample amplitude analysis result for the $\gamma p \rightarrow \pi^{+} \pi^{-} \pi^{+} n$ channel with GlueX. (top) The invariant mass spectrum as a function of $M\left(\pi^{+} \pi^{-} \pi^{+}\right)$is shown by the solid histogram. The results of the amplitude decomposition into resonant components in each bin is shown with points and error bars. (bottom) The exotic amplitude, generated at a relative strength of $1.6 \%$, is cleanly extracted (red points). The black points show the phase between the $\pi_{1}$ and $a_{1}$ amplitudes.

produced at the same rate as normal mesons, but the mock sample is designed to check sensitivity. The generated events were subjected to the full detector simulation and reconstruction. The result of the amplitude fit is shown in Figure 3, which indicates that with a pure sample of reconstructed decays, the GlueX detector provides excellent sensitivity to rare exotic decays. The analysis sensitivity will ultimately be limited by the ability to suppress and parametrize backgrounds in the amplitude analysis that arise from improperly reconstructed events.

\section{Status and Summary}

The civil construction of Hall D is complete and fabrication of all subsystems is underway. The magnet coils have been installed in Hall D and they are being connected up to the cryogenic supply and controls system in preparation for testing the complete magnet in the beginning of next year. The BCAL modules have been fabricated and will be outfitted with light guides and SiPM light sensors this fall so they can be installed into the bore of the magnet. The FCAL lead glass assemblies have been completed and the array will be stacked this fall. The construction of the drift chambers is well underway and they are on schedule for testing and installation in mid 2013. The construction of the tagger hodoscopes, time-of-flight and start counter systems will begin production this fall and are scheduled for installation after the drift chambers.

The GlueX experiment will take photoproduction data with a $9 \mathrm{GeV}$ linearly polarized photon beam in a region that is largely unexplored with the capability of measuring charged and neutral decays of mesons with masses up to about $2.5 \mathrm{GeV}$. The goal is to explore the production of hybrid mesons with sensitivities to signals that are a few percent of the total cross section. Commissioning of the experiment is scheduled for fall of 2014. 
Table 1. Compilation of hybrids with exotic quantum numbers, giving expected masses, widths, and decay predictions. Masses are estimated from dynamical LQCD calculations with $M_{\pi}=396 \mathrm{MeV} / c^{2}$ [2]. The PSS (Page, Swanson and Szczepaniak) and IKP (Isgur, Kokoski and Paton) model widths are from Ref. [18], with the IKP calculation based on the model in Ref. [7]. The total widths have a mass dependence, and Ref. [18] uses somewhat different mass values than suggested by the most recent lattice calculations [2]. Those final states marked with a dagger $(\dagger)$ are ideal for experimental exploration because there are relatively few stable particles in the final state or moderately narrow intermediate resonances that may reduce combinatoric background. (We consider $\eta, \eta^{\prime}$, and $\omega$ to be stable final state particles.)

\begin{tabular}{ccccccc}
\hline \hline & Approximate & $J^{P C}$ & \multicolumn{2}{c}{ Total Width (MeV) } & Relevant Decays & Final States \\
& Mass (MeV) & & PSS & IKP & & \\
\hline$\pi_{1}$ & 1900 & $1^{-+}$ & $80-170$ & 120 & $b_{1} \pi^{\dagger}, \rho \pi^{\dagger}, f_{1} \pi^{\dagger}, a_{1} \eta, \eta^{\prime} \pi^{\dagger}$ & $\omega \pi \pi^{\dagger}, 3 \pi^{\dagger}, 5 \pi, \eta 3 \pi^{\dagger}, \eta^{\prime} \pi^{\dagger}$ \\
$\eta_{1}$ & 2100 & $1^{-+}$ & $60-160$ & 110 & $a_{1} \pi, f_{1} \eta^{\dagger}, \pi(1300) \pi$ & $4 \pi, \eta 4 \pi, \eta \eta \pi \pi^{\dagger}$ \\
$\eta_{1}^{\prime}$ & 2300 & $1^{-+}$ & $100-220$ & 170 & $K_{1}(1400) K^{\dagger}, K_{1}(1270) K^{\dagger}, K^{*} K^{\dagger}$ & $K K \pi \pi^{\dagger}, K K \pi^{\dagger}, K K \omega^{\dagger}$ \\
\hline$b_{0}$ & 2400 & $0^{+-}$ & $250-430$ & 670 & $\pi(1300) \pi, h_{1} \pi$ & $4 \pi$ \\
$h_{0}$ & 2400 & $0^{+-}$ & $60-260$ & 90 & $b_{1} \pi^{\dagger}, h_{1} \eta, K(1460) K$ & $\omega \pi \pi^{\dagger}, \eta 3 \pi, K K \pi \pi$ \\
$h_{0}^{\prime}$ & 2500 & $0^{+-}$ & $260-490$ & 430 & $K(1460) K, K_{1}(1270) K^{\dagger}, h_{1} \eta$ & $K K \pi \pi^{\dagger}, \eta 3 \pi$ \\
\hline$b_{2}$ & 2500 & $2^{+-}$ & 10 & 250 & $a_{2} \pi^{\dagger}, a_{1} \pi, h_{1} \pi$ & $4 \pi, \eta \pi \pi^{\dagger}$ \\
$h_{2}$ & 2500 & $2^{+-}$ & 10 & 170 & $b_{1} \pi^{\dagger}, \rho \pi^{\dagger}$ & $\omega \pi \pi^{\dagger}, 3 \pi^{\dagger}$ \\
$h_{2}^{\prime}$ & 2600 & $2^{+-}$ & $10-20$ & 80 & $K_{1}(1400) K^{\dagger}, K_{1}(1270) K^{\dagger}, K_{2}^{*} K^{\dagger}$ & $K K \pi \pi^{\dagger}, K K \pi^{\dagger}$ \\
\hline \hline
\end{tabular}

\section{Acknowledgements}

We would like to especially thank Matt Shepherd for comments and suggestions in preparation of the talk and Jozef Dudek for patient explanations of the interpretation of lattice calculations. This work was supported by DOE Contract No. DE-AC05-06OR23177, under which Jefferson Science Associates, LLC, operates Jefferson Laboratory.

\section{References}

1. JLAB Experiment E12-06-102, (2006) http://www.jlab.org/exp_prog/proposals/06/PR12-06102.pdf

2. J.J. Dudek, Phys.Rev. D84, (2011) 074023

3. C.A. Meyer and Y. Van Haarlem, Phys.Rev. C82, (2010) 025208

4. J.J. Dudek et al., Phys.Rev.Lett. 103, (2009) 262001

5. J.J. Dudek et al., Phys.Rev. D82, (2010) 034508

6. J.J. Dudek et al., Phys.Rev., D83, (2011) 111502

7. N. Isgur et al., Phys.Rev.Lett. 54, (1985) 869

8. S.R. Cotanch and F.J. Llanes-Estrada, Nucl.Phys. A689, (2001) 481

9. P. Guo et al., Phys.Rev. D78, (2008) 056003

10. J.J. Dudek et al., Phys.Rev. D79, (2009) 094504

11. Y. Van Haarlem et al., Nucl.Instrum.Meth. A622, (2010) 142

12. B.D. Leverington et al., Nucl.Instrum.Meth. A596, (2008) 327

13. A. Brunner et al., Nucl.Instrum.Meth. A414, (1998) 466

14. G.S. Adams et al., Phys.Rev.Lett. 81, (1998) 5760

15. A.R. Dzierba et al., Phys.Rev. D73, (2006) 072001

16. M. Alekseev et al., Phys.Rev.Lett. 104, (2010) 241803

17. M. Nozar et al., Phys.Rev.Lett. 102, (2009) 102002

18. P.R. Page et al., Phys.Rev. D59, (1999) 034016 